\title{
Effect of aggregation form on bioavailability of zeaxanthin in humans: a randomised cross-over study
}

\author{
Judith Hempel $^{1}$, Anja Fischer ${ }^{1}$, Monique Fischer ${ }^{1}$, Josef Högel $^{2}$, Anja Bosy-Westphal ${ }^{3}$, Reinhold Carle B, $^{1,4}$ \\ and Ralf M. Schweiggert ${ }^{1 *}$ \\ ${ }^{1}$ Institute of Food Science and Biotechnology, University of Hohenheim, D-70599 Stuttgart, Germany \\ ${ }^{2}$ Institute of Human Genetics, University of Ulm, D-89081 Ulm, Germany \\ ${ }^{3}$ Institute of Nutritional Medicine, University of Hohenheim, D-70599 Stuttgart, Germany \\ ${ }^{4}$ Biological Science Department, King Abdulaziz University, SAU-21589 Jeddah, Saudi Arabia \\ (Submitted 14 April 2017 - Final revision received 3 September 2017 - Accepted 5 September 2017)
}

\begin{abstract}
Carotenoid bioavailability from plant and animal food is highly variable depending on numerous factors such as the physical deposition form of carotenoids. As the carotenoid zeaxanthin is believed to play an important role in eye and brain health, we sought to compare the human bioavailability of an $\mathrm{H}$-aggregated with that of a J-aggregated deposition form of zeaxanthin encapsulated into identical formulation matrices. A randomised two-way cross-over study with sixteen participants was designed to compare the post-prandial bioavailability of an $\mathrm{H}$-aggregated zeaxanthin and a J-aggregated zeaxanthin dipalmitate formulation, both delivering $10 \mathrm{mg}$ of free zeaxanthin. Carotenoid levels in TAG-rich lipoprotein fractions were analysed over $9.5 \mathrm{~h}$ after test meal consumption. Bioavailability from the J-aggregated formulation $(\mathrm{AUC}=55.9 \mathrm{nmol} \mathrm{h} / \mathrm{l})$ was $23 \%$ higher than from the H-aggregated one (AUC $=45.5 \mathrm{nmol} \mathrm{h} / \mathrm{l}$ ), although being only marginally significant $(P=0.064)$. Furthermore, the same formulations were subjected to an internationally recognised in vitro digestion protocol to reveal potential strengths and weaknesses of simulated digestions. In agreement with our human study, liberation of zeaxanthin from the J-aggregated formulation into the simulated duodenal fluids was superior to that from the H-aggregated form. However, micellization rate (bioaccessibility) of the J-aggregated zeaxanthin dipalmitate was lower than that of the $\mathrm{H}$-aggregated zeaxanthin, being contradictory to our in vivo results. An insufficient ester cleavage during simulated digestion was suggested to be the root cause for these observations. In brief, combining our in vitro and in vivo observations, the effect of the different aggregation forms on human bioavailability was lower than expected.
\end{abstract}

Key words: Carotenoids: Food matrix: H-aggregates: J-aggregates: Supplements

The bioavailability of carotenoids, that is, the fraction of ingested carotenoid that is absorbed to the blood circulation, depends on numerous factors such as the nutritional status of the host, co-consumption of lipids, food preparation and inherent traits of the food matrix. Although the effect of co-consumed lipids has been widely studied ${ }^{(1-4)}$, the influence of the natural deposition form of carotenoids in plant and animal foods merits deeper investigation, since it was suggested to substantially influence carotenoid bioavailability. For instance, bioavailability of a presumably liquid-crystalline deposition form of $\beta$-carotene from tubular chromoplasts of papaya was shown to be higher than that of solid-crystalline $\beta$-carotene from carrot ${ }^{(5)}$. Although the physical deposition form of the carotenoids present in papaya and other foods with tubular chromoplasts is believed to represent a liquid-crystalline state, direct evidence is still lacking. Furthermore, the comparison of bioavailability from different plant foods has been inconclusive for determining the sole effect of the carotenoid deposition form, mainly because of the interfering influence of other food matrix constituents and properties, such as the more rigid cells walls of carrots when compared with those of papaya.

Therefore, in the present study, we aimed at investigating the influence of two different carotenoid deposition forms from identical food matrices. Because of its nutritional importance and its ability to form different aggregates, zeaxanthin was selected as a model carotenoid in this study. Particularly in ocular tissues, zeaxanthin and its structural isomers lutein and meso-zeaxanthin are supposed to act as protective short wavelength (blue) light filters and to exert antioxidant and antiinflammatory properties ${ }^{(6,7)}$. In agreement with these protective functions, a diet rich in lutein and zeaxanthin was associated with delayed onset of several eye-related diseases such as agerelated macular degeneration or cataract ${ }^{(8)}$. Driven by the formation of hydrogen bonds, zeaxanthin was earlier shown to form tightly packed, so-called H-aggregates. When the formation of hydrogen bonds was prevented by, for example,

Abbreviations: MTBE, methyl tert-butyl ether; TRL, TAG-rich lipoprotein.

* Corresponding author: Dr R. M. Schweiggert, fax +49 711459 24110, email ralf.schweiggert@uni-hohenheim.de 
esterification of the hydroxyl groups, zeaxanthin has been observed to form more loosely packed, so-called J-aggregates ${ }^{(\mathcal{(}, 10)}$. Such J-aggregates of carotenoid esters have been hypothesised to inherently form a liquid-crystalline phase similar to that found in the tubular chromoplasts of several plant foods such as papaya ${ }^{(11)}$ or goji berries ${ }^{(12)}$.

In this study, we produced two formulations with differently aggregated zeaxanthin to investigate the effect of different carotenoid deposition forms on carotenoid bioavailability in humans. Using the same encapsulation matrix to minimise matrix differences, one formulation contained $\mathrm{H}$-aggregated zeaxanthin and one formulation contained J-aggregated zeaxanthin dipalmitate. The bioavailability of these different zeaxanthin forms was then compared in a randomised single-blind, cross-over human intervention study. As direct comparisons of human bioavailability to in vitro bioaccessibility data are rare, we subjected the test foods used in the human study to a concurrent in vitro digestion procedure following the standardised INFOGEST model in order to reveal strengths and weaknesses of such frequently used digestion models.

\section{Methods}

\section{Materials}

Authentic standards of (all- $E, 3 R, 3^{\prime} R$ )-zeaxanthin and (all-E, $3 R, 3^{\prime} R$ )-zeaxanthin dipalmitate were purchased from Biomol and CaroteNature, respectively. Dried, organically grown goji berries were obtained from PÄX Food. Modified food starch (Purity Gum 2000) was kindly donated from Ingredion. Chemicals and solvents used for the production of carotenoid formulations were of pharmaceutical grade (Ph. Eur.), and other solvents and chemicals were of analytical or HPLC grade.

\section{Participants}

A total of sixteen healthy, non-pregnant and non-smoking human subjects (eight male and eight female) aged 21-30 years (median 25) with a BMI of $22 \cdot 8(\mathrm{SD} 2 \cdot 1) \mathrm{kg} / \mathrm{m}^{2}$ participated in the study. For a significance level of $\alpha=0.05$, a paired $t$ test indicated that an enrolment of sixteen combined men and women will provide $98 \%$ power for detecting the anticipated difference in bioavailability of at least $25 \%(\sigma=0 \cdot 25)$, regarding the primary endpoints of AUC TAG-rich lipoprotein (TRL) zeaxanthin responses. The eligibility of the participants was verified by a health and lifestyle questionnaire. None of the participants suffered from cancer, chronic gastrointestinal disease or took drugs lowering plasma TAG or cholesterol concentrations. Regular use of nutritional supplements containing carotenoids and frequent alcohol consumption were further exclusion criteria. Further characteristics of the participants (fasting plasma concentrations of total TAG and cholesterol) are shown in Table 1 . This study was conducted according to the guidelines laid down in the Declaration of Helsinki, and all procedures involving patients were approved by the Ethics Committee of the Medical Association of the Federal State of Baden-Württemberg (Stuttgart, Germany, project no. F-2015-015). Signed informed consent was obtained from all patients.
Table 1. Characteristics of the study participants (Mean values and standard deviations)

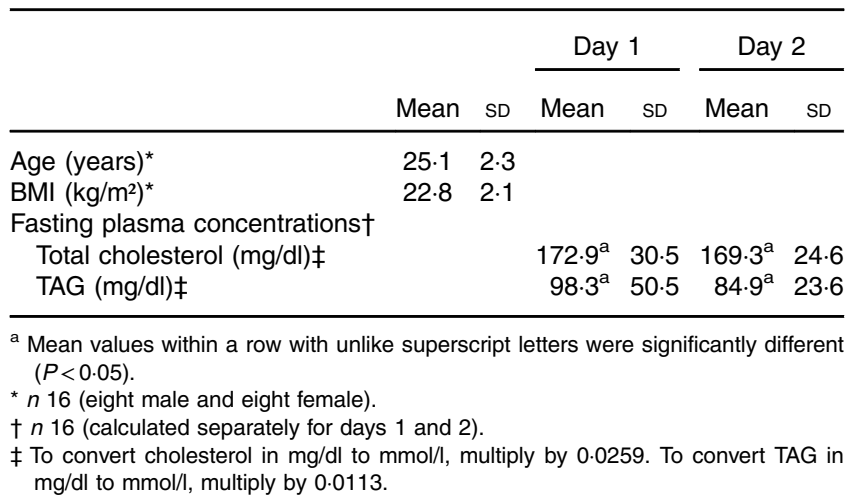

\section{Study design}

The study was designed as a randomized single-blind (participant only), two-way cross-over study and was conducted at the University of Hohenheim between May and July 2016. The cross-over study design was chosen over a parallel study design owing to previously observed high inter-individual differences in carotenoid bioavailability $^{(13,14)}$. On an initial visit, the clinical procedures were explained to the participants, and age and BMI $\left(\mathrm{kg} / \mathrm{m}^{2}\right)$ were recorded. All participants were asked to follow a diet low on carotenoids for $14 \mathrm{~d}$ before each of their day-long clinical visits ('washout'). A list of carotenoid-containing foods to avoid was provided to all participants at the initial visit. Foods to be avoided were selected according to the US Department of Agriculture National Nutrient Database for Standard Reference, release $28^{(15)}$. Following a randomised cross-over design, each participant consumed the zeaxanthin and zeaxanthin dipalmitate formulation once.

After fasting overnight for at least $12 \mathrm{~h}$, participants arrived at the Institute of Nutritional Medicine (University of Hohenheim) for drawing a baseline blood sample $(0 \mathrm{~h})$ via catheter from their forearm vein. Subsequently, participants received a balanced breakfast containing rice flakes (80 g), water (150 g), sugar (10 g), low-fat yogurt (136g), soya oil (14 g), frozen raspberries $(50 \mathrm{~g})$ and an optional cup of coffee together with the respective formulation providing $10 \mathrm{mg}(=17.6 \mu \mathrm{mol})$ of zeaxanthin, which had been dispersed in $80 \mathrm{~g}$ of apple juice and $80 \mathrm{~g}$ of apple puree for $1 \mathrm{~min}$ using a conventional blender (Kult X Mix\&Go, 300 W; WMF). Test meal formulations were served in coloured non-transparent cups to ensure a similar test meal appearance. All participants were asked to consume the breakfast within $20 \mathrm{~min}$. The total energetic value of the breakfast was $2610 \mathrm{~kJ}$, provided by $16 \mathrm{~g}$ of fat, $13 \mathrm{~g}$ of protein, $99 \mathrm{~g}$ of carbohydrates and $2 \mathrm{~g}$ of dietary fibre. Further blood samples were drawn after 2, 3, 4, 5, 6, 8 and $9.5 \mathrm{~h}$. At $4.5 \mathrm{~h}$, a carotenoid-free pizza-like dish (without tomato sauce) and a chocolate pudding providing $3619 \mathrm{~kJ}$ through $38 \mathrm{~g}$ of fat, $27 \mathrm{~g}$ of protein and $103 \mathrm{~g}$ of carbohydrates was served in order to facilitate a further release of carotenoids temporarily held back in the enterocytes after digestion of the preceding test meal. Food composition data were derived from the values given in the nutrition facts labels on the packing of the respective 
commercial foods. Participants were allowed to consume water ad libitum. No further drinks or snacks were permitted during their stay. After 2 weeks of a second washout period, participants returned to the institute for their second day-long visit to consume the respective test meal they did not consume before. The order in which participants consumed test meals (zeaxanthin test meal: A; zeaxanthin dipalmitate test meal: B) was randomised. Each test meal order (AB and BA) was followed by eight participants randomly selected from the total of sixteen participants. No adverse effects were observed and all participants completed the study.

\section{Blood sampling and carotenoid extraction from TAG-rich lipoprotein samples}

Blood samples (approximately $12 \mathrm{ml}$ ) were drawn from a forearm vein into $\mathrm{K}_{2}$ EDTA tubes (S-Monovette ${ }^{\circledR}$ EDTA/ $\mathrm{K}_{2}$-Gel; Sarstedt) and plasma was separated by centrifugation (2300 $\boldsymbol{g}$ ) for $10 \mathrm{~min}$ at $4^{\circ} \mathrm{C}$. Isolation of TRL fractions from fresh (unfrozen) plasma and carotenoid analysis of TRL fractions was performed according to a method by Kopec et $a l .^{(16)}$. In brief, $2.5 \mathrm{ml}$ of plasma was transferred into polyallomer tubes and $0.8 \mathrm{~g}$ of potassium bromide was added and dissolved under stirring. The mixture was overlayered with $1 \mathrm{ml}$ of a sodium chloride solution $(1.006 \mathrm{~kg}$ per 1) and subjected to ultracentrifugation $\left(40700 \mathrm{rpm} \approx 154920 \mathrm{~g}\right.$ ) at $20^{\circ} \mathrm{C}$ for $33 \mathrm{~min}$. After centrifugation, the lower plasma fraction was drained from the tube and the upper TRL-containing fraction (approximately $0.5 \mathrm{ml}$ ) was collected in a cryovial. Subsequently, the tube was rinsed twice with each $0.25 \mathrm{ml}$ of the above-mentioned sodium chloride solution, which was added to the TRL fraction. For extraction, $0.5 \mathrm{ml}$ of TRL fraction and $0.5 \mathrm{ml}$ of ethanol were mixed on a vortex for $10 \mathrm{~s}$. Subsequently, $2 \mathrm{ml}$ of hexane was added and the mixture was probe-sonicated (Sonopuls HD 3100 with MS 72 sonotrode; Bandelin) three times at $75 \%$ amplitude for $8 \mathrm{~s}$ and subsequently centrifuged (966 g) for $3 \mathrm{~min}$. The upper organic phase was separated and the lower phase was re-extracted using $2 \mathrm{ml}$ of hexane. The combined organic phases were evaporated to dryness by a gentle stream of $\mathrm{N}_{2}$ and stored at $-80^{\circ} \mathrm{C}$. Before HPLC analysis, samples were re-dissolved in $100 \mu \mathrm{l}$ of methyl tert-butyl ether (MTBE) and $100 \mu \mathrm{l}$ of methanol and membrane-filtered into amber glass vials. Carotenoid identification and quantification were performed according to a method described by Kopec et al. ${ }^{(16)}$. Zeaxanthin was quantified using an external calibration curve of an authentic zeaxanthin standard. Zeaxanthin dipalmitate, which had not previously been investigated by Kopec et al. ${ }^{(16)}$, was identified using an authentic zeaxanthin dipalmitate standard.

\section{Preparation of zeaxanthin formulations}

Extraction of carotenoids from goji berries. Carotenoid extraction was performed with explosion-proof devices of the pilot plant of the Fraunhofer Institute for Process Engineering and Packaging IVV in Freising, Germany. Approximately $10 \mathrm{~kg}$ of dried goji berries were ground using a mortar, and seeds were removed using a vibratory sieve shaker (ZM1; Retsch). The obtained ground powder was extracted with 100 litres of hexane and 20 litres of methanol in a double-walled explosionproof extraction vessel (capacity 300 litres) with a wall temperature of $40^{\circ} \mathrm{C}$ under stirring and forced solvent circulation for $2 \mathrm{~h}$. Subsequently, the extraction solvent containing the extracted lipophilic components was pumped through a filter candle (polypropylene, 20", $1 \mu \mathrm{m}$ pore size; MTS \& APIC Filter) into a second double-walled vessel (capacity 300 litres), where the extract was concentrated to approximately 10 litres under vacuum (300 mbar) and forced circulation at a wall temperature of $40^{\circ} \mathrm{C}$. After concentration to dryness using a rotary evaporator $\left(40^{\circ} \mathrm{C}\right)$, the concentrated primary extract was obtained.

For purification from further non-carotenoid constituents, the concentrated primary extract was washed with ethanol, re-dissolved in hexane and washed with water. Subsequently, the hexane phase was evaporated to dryness. The carotenoid composition of the final extract was mainly composed of (all-E)zeaxanthin dipalmitate (approximately $90 \%$ of HPLC peak area at $450 \mathrm{~nm}$ ).

Saponification. Saponification of the above-described concentrated primary extract from goji berry was carried out according to Khachik ${ }^{(17)}$ with some modifications. An aliquot of $900 \mathrm{mg}$ of extract was dissolved in $300 \mathrm{ml}$ of diethyl ether in an amber glass bottle $(500 \mathrm{ml}), 100 \mathrm{ml}$ of ethanolic $\mathrm{KOH}(10 \%, \mathrm{w} / \mathrm{v})$ was added, the bottle was flushed with $\mathrm{N}_{2}$ and the mixture was incubated at room temperature under continuous stirring overnight. Successful saponification was confirmed by TLC on silica gel plates (TLC Silica gel 60 F254; Merck) using hexane/ethyl acetate $(70: 30, \mathrm{v} / \mathrm{v})$ as mobile phase. Subsequently, the formed soapstock was removed by filtration (MN 615, 4-12 $\mu \mathrm{m}$; Macherey-Nagel), the $\mathrm{pH}$ of the filtered mixture was decreased to 7 (SD 1) using $2.5 \mathrm{~m}$ hydrochloric acid and all solvents were removed by rotary evaporation. The carotenoid composition of the saponified extract predominantly contained (all-E)-zeaxanthin (approximately $90 \%$ of HPLC peak area at $450 \mathrm{~nm}$ ).

Production of carotenoid formulations. The above-described final extract and the saponified extract derived thereof were used for the production of zeaxanthin dipalmitate and zeaxanthin formulations, respectively. The respective extract was dissolved in acetone and the concentration of the solution was determined photometrically using the molar absorption coefficient of zeaxanthin in acetone $\left(\varepsilon_{452 \mathrm{nn}}=132900\right.$ litre/ $\mathrm{mol} \cdot$ per $\left.\mathrm{cm}^{(18)}\right)$. After adjusting the concentration to $2 \cdot 10^{-4} \mathrm{~mol} / \mathrm{l}$, aliquots of $300 \mathrm{ml}$ of the respective acetonic solution were separated delivering $6 \cdot 10^{-5} \mathrm{~mol}$ zeaxanthin $(34 \cdot 1 \mathrm{mg})$ or $6 \cdot 10^{-5} \mathrm{~mol}$ zeaxanthin dipalmitate $(62.7 \mathrm{mg})$. Each $300-\mathrm{ml}$ aliquot was supplemented with $6.8 \mathrm{mg}$ of $\alpha$-tocopherol and then subjected to $700 \mathrm{ml}$ of a water phase containing $6.8 \mathrm{mg}$ of ascorbic acid, $109 \mathrm{mg}$ of gum arabic, $109 \mathrm{mg}$ of glucose, $171 \mathrm{mg}$ of modified starch and $246 \mathrm{mg}$ of maltodextrin under $\mathrm{N}_{2}$ flushing and vigorous stirring using an Ultra Turrax (IKA-Werke). Subsequently, the acetone was completely removed from the mixture by rotary evaporation and the aqueous formulation was freeze-dried (VaCo 10-II-E; Zirbus). The resulting powders were 
filled into amber glassware, flushed with $\mathrm{N}_{2}$ and stored at $-80^{\circ} \mathrm{C}$ until use.

\section{Characterisation of carotenoid content and aggregate type of zeaxanthin formulations}

Aggregate type. An aliquot of $50 \mathrm{mg}$ formulation was dispersed in distilled water and filtered (MN 615 1/4, 4-12 $\mu \mathrm{m}$ ) to remove eventual large agglomerates to minimise signal loss due to scattering and reflection. Then, the UV visible (UV/Vis) absorption spectrum was measured in the range of $300-600 \mathrm{~nm}$ using a spectrophotometer (Lambda 35; Perkin Elmer).

Carotenoid content. An aliquot of $5 \mathrm{mg}$ of formulation was dispersed in $1.5 \mathrm{ml}$ of salivary solution $(4.3 \% \mathrm{w} / \mathrm{v} \mathrm{CaCl} 2,19.5 \%$ $\mathrm{w} / \mathrm{v} \mathrm{KH}_{2} \mathrm{PO}_{4}, 28.6 \% \mathrm{w} / \mathrm{v} \mathrm{NaCl}, 47.7 \% \mathrm{w} / \mathrm{v} \mathrm{NaHCO} 3$ in water, $\mathrm{pH} 7), 1.5 \mathrm{ml}$ of ultrapure water and $200 \mu \mathrm{l}$ of an amylase solution (11.8 mg $\alpha$-amylase in $1 \mathrm{ml}$ phosphate buffer $(\mathrm{pH} 7$, $3.575 \mathrm{mg} / \mathrm{ml} \mathrm{Na}_{2} \mathrm{HPO}_{4} .2 \mathrm{H}_{2} \mathrm{O}$ in water). The mixture was incubated at room temperature under continuous stirring for $1 \mathrm{~h}$. Subsequently, carotenoid extraction and separation via HPLC-diode array detection (DAD) analysis was performed as described by Hempel et l $^{(12)}$. In brief, $2 \mathrm{ml}$ of extraction solvent (methanol/ethyl acetate/light petroleum (1:1:1, v/v/v) containing $0.1 \mathrm{~g} / \mathrm{l}$ butylhydroxyanisole and $0.1 \mathrm{~g} / \mathrm{l}$ butylhydroxytoluene) was added and the mixture was probe-sonicated at $70 \%$ amplitude for $20 \mathrm{~s}$. After centrifugation (1300 $\mathrm{g}$ ) for 3 min, the upper organic phase was separated and the extraction procedure was repeated two to three times until the organic phase was colourless. The combined organic phases were evaporated to dryness by a gentle stream of $\mathrm{N}_{2}$ and stored at $-80^{\circ} \mathrm{C}$. Before HPLC analyses, the dried extracts were made up in $3 \mathrm{ml}$ of a binary mixture of MTBE and methanol (1:1 for samples containing zeaxanthin, 9:1 for samples containing zeaxanthin dipalmitate). Separation was performed on a Waters 2695 separations module equipped with a 2996 photodiode array detector (Waters). Carotenoids were eluted on a C30 reversed-phase column $(150 \times 3 \mathrm{~mm}$ i.d., $3 \mu \mathrm{m}$ pore size; YMC Europe) protected by a guard column of the same material. For zeaxanthin analysis, eluent $\mathrm{A}(=90: 10(\mathrm{v} / \mathrm{v})$ methanol-water) and eluent B $(=20: 78: 2(\mathrm{v} / \mathrm{v} / \mathrm{v})$ methanol-MTBE-water $)$ were used in a gradient programme described by Hempel et al ${ }^{(9)}$. For zeaxanthin dipalmitate analysis, eluent A $(=80: 18: 2(\mathrm{v} / \mathrm{v} / \mathrm{v})$ methanol-MTBEwater $)$ and eluent B $(=8: 90: 2(\mathrm{v} / \mathrm{v} / \mathrm{v})$ methanol-MTBE-water $)$ were used in a gradient programme described by Hempel et al. ${ }^{(12)}$. All eluents contained ammonium acetate $(0.4 \mathrm{~g} / 1$ solvent). For quantification, linear calibration curves of authentic standards of zeaxanthin and zeaxanthin dipalmitate were used. Storage stability was tested over a period of $154 \mathrm{~d}$ at $-80^{\circ} \mathrm{C}$ under light exclusion. All measurements were performed in triplicate.

\section{In vitro bioaccessibility of carotenoids}

In vitro bioaccessibility of the zeaxanthin formulations was performed following the INFOGEST in vitro digestion protocol $^{(19)}$, as described in detail by Hempel et al. ${ }^{(12)}$ with the following slight modification. Being omitted in the original protocol, an aliquot of $5 \mathrm{U}$ of cholesterol esterase (SigmaAldrich Chemie) was added to the digesta at the start of the intestinal phase as previously proposed by Schweiggert et $a l^{(20)}$. The test meal included $35 \mathrm{mg}$ of the respective formulation containing either $\mathrm{H}$-aggregated zeaxanthin or J-aggregated zeaxanthin dipalmitate $(1.8 \mu \mathrm{mol}$ zeaxanthin or $1.3 \mu$ mol zeaxanthin dipalmitate) being combined with $1.21 \mathrm{~g}$ of low-fat yogurt, $0.04 \mathrm{~g}$ of soya oil, $0.67 \mathrm{~g}$ of rice flakes, $1.25 \mathrm{~g}$ of water, $0.08 \mathrm{~g}$ of sugar, $0.42 \mathrm{~g}$ of raspberries, $0.67 \mathrm{~g}$ of apple puree and $0.67 \mathrm{~g}$ of apple juice in order to widely mimic the composition of the test meal fed during the human studies. Simulated digestion fluids were prepared as described for the INFOGEST model by Minekus et al. ${ }^{(19)}$. The in vitro digestion comprised an oral phase, a gastric phase and an intestinal phase. After completion of the in vitro digestion, carotenoid levels in the digesta were analysed as described by Hempel et $a l .{ }^{(12)}$. In brief, samples were made up to a defined volume $(50 \mathrm{ml})$ and centrifuged $(75000 \mathrm{~g})$ for $60 \mathrm{~min}$. After centrifugation, an aliquot of the supernatant was directly frozen at $-80^{\circ} \mathrm{C}$, whereas the remainder was membrane-filtered $(0.2 \mu \mathrm{m}$, cellulose acetate) and then stored at $-80^{\circ} \mathrm{C}$. For extraction, an aliquot of $2 \mathrm{ml}$ of unfiltered or filtered digesta was combined with $2 \mathrm{ml}$ of extraction solvent (methanol-ethyl acetate-light petroleum $(1: 1: 1, \mathrm{v} / \mathrm{v} / \mathrm{v})$ containing $0 \cdot 1 \mathrm{~g} / \mathrm{l}$ butylhydroxyanisole and $0 \cdot 1 \mathrm{~g} / \mathrm{l}$ butylhydroxytoluene) and extracted using a probe sonicator as described in the "Characterisation of carotenoid content and aggregate type of zeaxanthin formulations' section. Extraction was repeated two times until the organic phase was colourless. The combined organic phases were evaporated to dryness by a gentle stream of $\mathrm{N}_{2}$ and stored at $-80^{\circ} \mathrm{C}$. Before HPLC analysis, samples were made up in $0.5 \mathrm{ml}$ (filtered digesta) or $1 \mathrm{ml}$ (unfiltered digesta) of a binary mixture of MTBE and methanol and analysed as described in the 'Characterisation of carotenoid content and aggregate type of zeaxanthin formulations' section. Carotenoid 'liberation' refers to the percentage of the initially fed carotenoid dose that had been transferred to the supernatant obtained after centrifugation of the digesta derived after completing the intestinal phase. Carotenoid 'bioaccessibility' refers to the percentage of the initially fed carotenoid dose that had been transferred to the micellar phase, which was obtained by membrane filtration of the above-mentioned supernatant. Both the digestions and the respective carotenoid analyses were performed in triplicate.

\section{Statistics}

The Shapiro-Wilk test for normality $(P<0.05)$ was performed and significant differences between means of bioaccessibility data and TAG and cholesterol data of blood samples were assessed by means of independent $t$ tests $(P<0.05)$ using SAS University Edition (SAS Institute).

The baseline-corrected area under the concentration $v$. time curve (AUC) was determined by trapezoidal approximation using the respective concentration and time data points over 9.5 h (Excel 2013; Microsoft Corporation). As previously described $^{(5,21)}$, the AUC was used as a representative parameter for comparing post-prandial bioavailability. An ANOVA was carried out to model AUC values using a linear mixed model 
with the covariates food sequence (two combinations), period (two clinical visits), participants ( $n$ 16) and formulation (zeaxanthin, zeaxanthin dipalmitate). In addition, the non-parametric Cochran-Mantel-Haenszel test was used with the different food sequences as strata for the overall and pairwise identification of significant differences between means, controlling for carryover, period and time effects. $P$ values quoted in the text are from ANOVA unless stated otherwise. Data were analysed using SAS 9.3 (SAS Institute).

\section{Results}

\section{Characterisation of zeaxanthin formulations}

According to Hempel et al. ${ }^{(9)}$, free zeaxanthin forms H-aggregates and esterified zeaxanthin forms $\mathrm{J}$-aggregates in hydrated polar solvents such as acetone/water mixtures, being easily identified by the presence of a hypsochromic (H-aggregate) or a bathochromic absorption band (J-aggregate) in the respective UV/Vis spectra. For better illustration of these aggregate archetypes, the absorption spectra of an aggregated standard of zeaxanthin and zeaxanthin dipalmitate in acetone/water (20:80, v/v) are depicted in Fig. 1. The absorption band at approximately 380-400 nm of the aggregates of the formulation containing free zeaxanthin (Fig. 1(a), — ) resembled that of the H-aggregated zeaxanthin standard (Fig. 1(a), ----), clearly revealing the presence of $\mathrm{H}$-aggregated zeaxanthin. The aggregates of the formulation containing zeaxanthin dipalmitate produced a substantially different UV/Vis absorption spectrum (Fig. 1(b)) as compared with the H-aggregates. Although the fine structure of the UV/Vis absorption spectrum of the zeaxanthin dipalmitate aggregates was lost upon formulation (Fig. 1(b), —), the absorption maximum at approximately $450 \mathrm{~nm}$ and the vast broadening of the spectrum suggests the presence of J-aggregated zeaxanthin in the used formulation.

Throughout the whole intervention study, carotenoid contents of both zeaxanthin formulations did not show significant degradation. At a storage temperature of $-80^{\circ} \mathrm{C}$ under light exclusion, the H-aggregated formulation showed $95 \%$ of zeaxanthin retention after $122 \mathrm{~d}$ of storage. Slight carotenoid degradation was observed after $154 \mathrm{~d}$ of storage $(87 \%$ of zeaxanthin retention). The J-aggregated formulation showed $94 \%$ of zeaxanthin dipalmitate retention after $78 \mathrm{~d}$ of storage. After 122 and $154 \mathrm{~d}$ of storage, a slight degradation (89\% of zeaxanthin dipalmitate retention) was observed.

\section{Zeaxanthin response in TAG-rich lipoprotein fractions}

The mean baseline-corrected concentration $v$. time curve of zeaxanthin in the TRL fractions is shown in Fig. 2. Maximum zeaxanthin levels in TRL fractions were observed at $6 \mathrm{~h}$ after test meal consumption, irrespective of the ingested formulation. Consumption of the test meal containing the J-aggregated zeaxanthin dipalmitate led to a $20 \%$ higher maximum zeaxanthin concentration (13.80 (SD 2.14) nmol/l) as compared with that containing the $\mathrm{H}$-aggregated free zeaxanthin $(10.66$ (SD 1.87) nmol/l). Similarly, the mean baseline-corrected AUC was $23 \%$ higher when the participants had consumed the

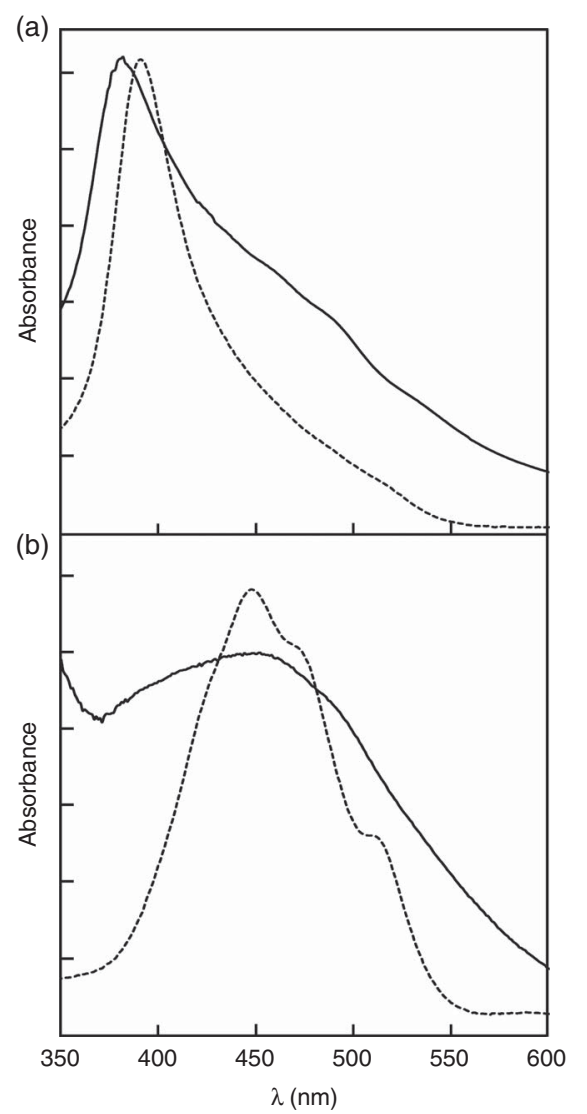

Fig. 1. UV visible (UV/Vis) absorption spectra of the (a) $\mathrm{H}$-aggregated zeaxanthin and (b) J-aggregated zeaxanthin dipalmitate formulation. UV/Vis absorption of the formulations dispersed in water. ----, UV/Vis absorption of the respective aggregated pure compound. Aggregates were produced by precipitation upon addition of water to an acetonic solution of the respective pure compound (acetone:water ratio, 2:8, v/v).

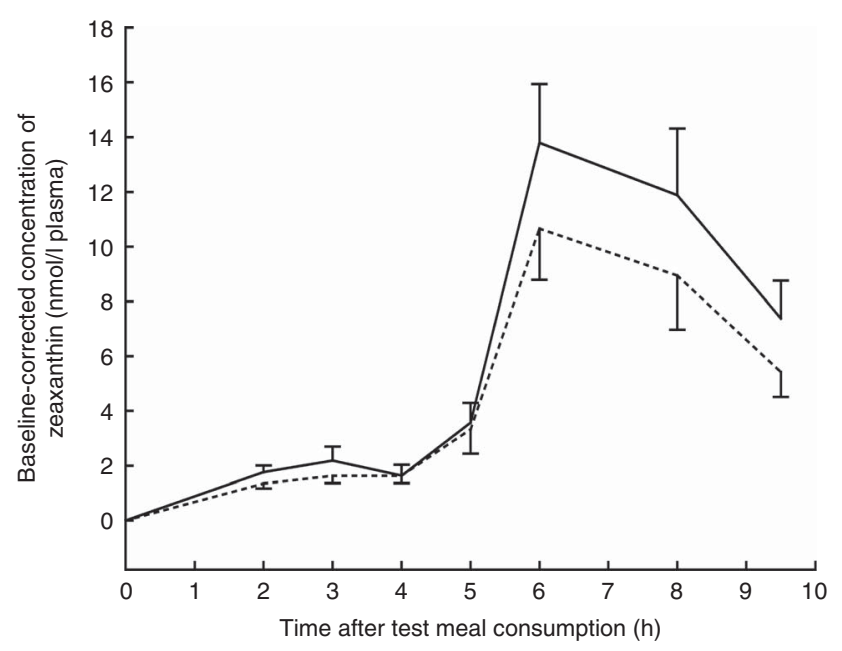

Fig. 2. Baseline-corrected concentrations of zeaxanthin in the TAG-rich lipoprotein fractions after consumption of the zeaxanthin (----) or zeaxanthin dipalmitate formulation $(-)$. Values are means $(n 16)$, with their standard errors represented by vertical bars. —, J-aggregated zeaxanthin dipalmitate formulation; ----, H-aggregated zeaxanthin formulation. Mean AUC after consumption of the J-aggregated zeaxanthin dipalmitate formulation was $23 \%$ higher than after consumption of the $\mathrm{H}$-aggregated zeaxanthin formulation, however, only reaching marginal statistical significance $(P=0.064)$. 


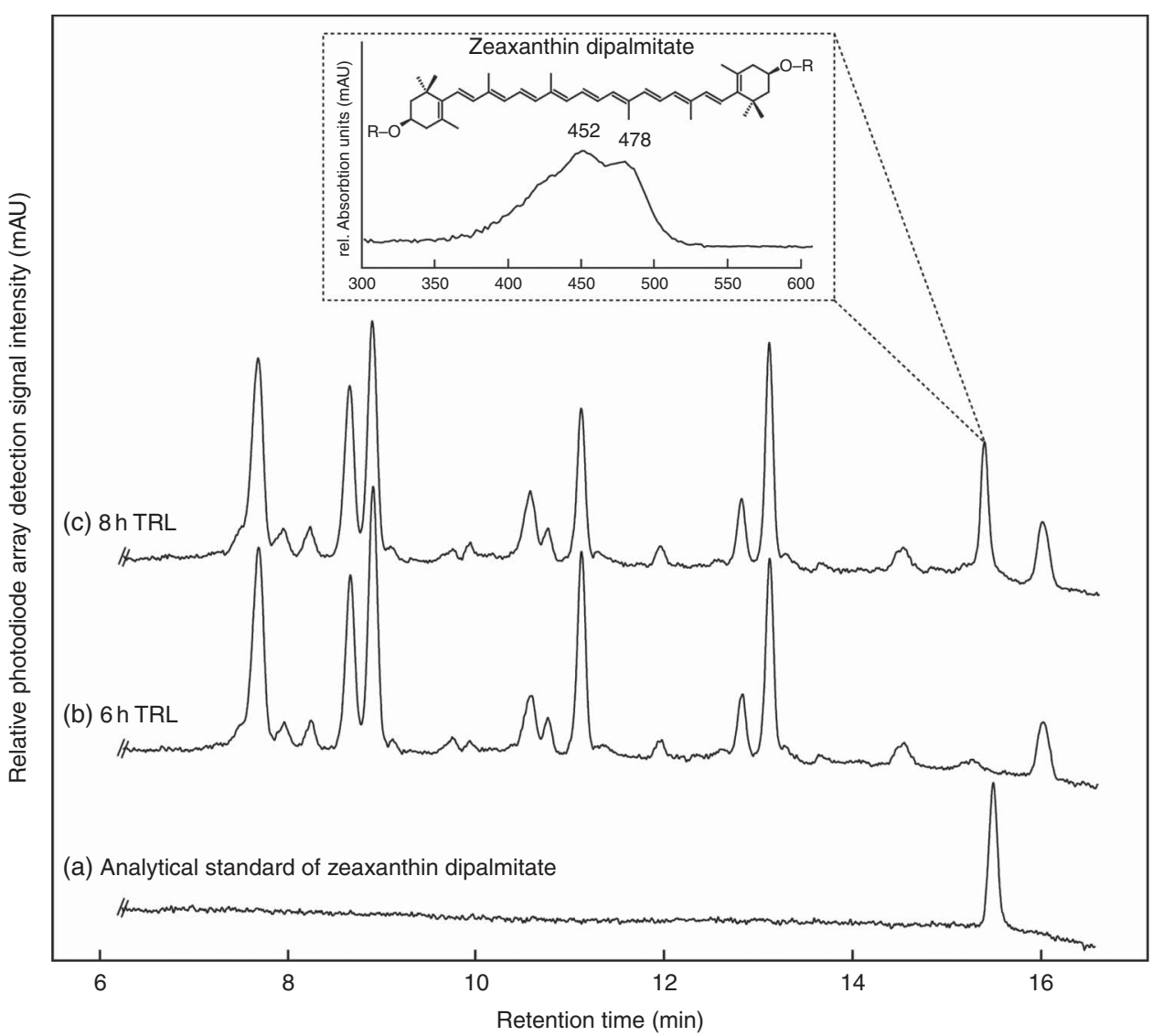

Fig. 3. HPLC separation of an (a) analytical zeaxanthin dipalmitate standard and carotenoids from human TAG-rich lipoprotein (TRL) fractions of one participant at (b) 6 and (c) $8 \mathrm{~h}$ after consumption of J-aggregated zeaxanthin dipalmitate monitored at $450 \mathrm{~nm}$. The detail shows the UV visible spectrum of the new putative zeaxanthin dipalmitate peak occurring post prandium. The putative zeaxanthin dipalmitate was observed on several instances $(1 \times$ at $2 \mathrm{~h}, 2 \times$ at $3 \mathrm{~h}, 1 \times$ at $4 \mathrm{~h}, 1 \times$ at $6 \mathrm{~h}$ and $4 \times$ at $8 \mathrm{~h}$ ), and also after the consumption of the $\mathrm{H}$-aggregated 'free' zeaxanthin.

J-aggregated zeaxanthin dipalmitate (55.89 (sD 36.35) nmol h/l) than when they had consumed the H-aggregated free zeaxanthin (45.50 (SD 32.99) nmol h/l), however, being only marginally significant $(P=0.064)$. Moreover, the median AUC was $49 \%$ higher for the J-aggregated formulation ( $49.72 \mathrm{nmol} \mathrm{h} / \mathrm{l})$ as compared with the H-aggregated formulation $(33.30 \mathrm{nmol} \mathrm{h} / \mathrm{l})$.

Regardless of the intake of esterified or free zeaxanthin, only free zeaxanthin was observed in most TRL fractions with some unexpected exceptions. In nine out of thirty-two TRL sample sets (sixteen test persons, 2 intervention d), the apparent presence of zeaxanthin dipalmitate was observed at single time points (see Fig. 3). In five out of the above-mentioned nine occurrences of zeaxanthin dipalmitate in the TRL, test persons had consumed the powder containing only free zeaxanthin. The presence of zeaxanthin dipalmitate in TRL fractions usually occurred only at a single time point, however, not always at the same hour for each participant $(1 \times$ at $2 \mathrm{~h}, 2 \times$ at $3 \mathrm{~h}, 1 \times$ at $4 \mathrm{~h}$, $1 \times$ at $6 \mathrm{~h}$ and $4 \times$ at $8 \mathrm{~h}$ ).

\section{In vitro zeaxanthin bioaccessibility}

The results of the in vitro digestion are shown in Fig. 4. Carotenoid liberation from the J-aggregated zeaxanthin dipalmitate formulation into the simulated duodenal fluid (64.0 (SD 3.5)\%) was nearly twice as high as from the $\mathrm{H}$-aggregated formulation

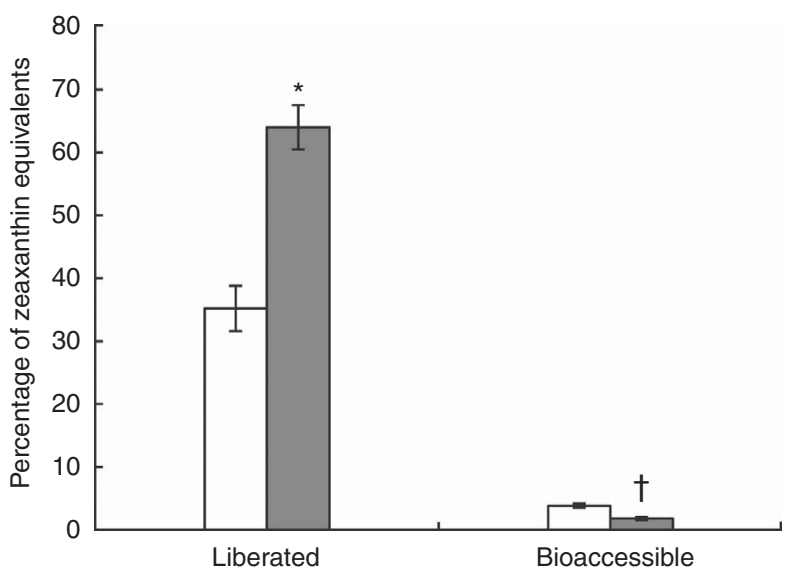

Fig. 4. Percentage of zeaxanthin equivalents in centrifuged digesta (liberated), and centrifuged and filtrated $(0.2 \mu \mathrm{m})$ digesta (bioaccessible), after in vitro digestion of the $\mathrm{H}$-aggregated zeaxanthin $(\square)$ and $\mathrm{J}$-aggregated zeaxanthin dipalmitate $(\square)$ formulation. Values are means $(n 3)$, with standard deviations represented by vertical bars. * Liberation of J-aggregated zeaxanthin dipalmitate was significantly higher than that of $\mathrm{H}$-aggregated zeaxanthin $(P<0.05)$. $\dagger$ Bioaccessibility of $\mathrm{J}$-aggregated zeaxanthin dipalmitate was significantly lower than that of $\mathrm{H}$-aggregated zeaxanthin $(P<0.05)$.

(35.2 (sD 3.6)\%), showing a significant difference $(P<0.05)$. When digesting the J-aggregated zeaxanthin dipalmitate formulation derived from the goji berry extract, similar 
proportions of zeaxanthin dipalmitate ( $83 \%$ of HPLC peak area at $450 \mathrm{~nm})$ and free zeaxanthin (1\%; as naturally contained in goji berries) were determined in test meal and simulated duodenal fluid. Although a high percentage of zeaxanthin equivalents was liberated from both formulations (33-67\%), the micellar fraction contained only a small percentage of zeaxanthin equivalents (2-4\%). In contrast to zeaxanthin liberation, zeaxanthin bioaccessibility was significantly higher from the $\mathrm{H}$-aggregated zeaxanthin formulation than from the J-aggregated zeaxanthin dipalmitate formulation $(3.8$ (SD 0.3$) v .1 \cdot 8$ (SD 0.2$) \%$, $P<0.05)$. Noteworthy, the micellar fraction after digestion of the J-aggregated zeaxanthin dipalmitate formulation contained higher percentages of free zeaxanthin ( $7 \%$ of HPLC peak area at $450 \mathrm{~nm}$ ) and lower percentages of zeaxanthin dipalmitate $(52 \%)$, as found in the whole simulated duodenal fluid.

\section{Discussion}

\section{Bioavailability of zeaxanthin aggregates}

The post-prandial bioavailability of zeaxanthin from the J-aggregated zeaxanthin dipalmitate formulation was $23 \%$ higher than from the H-aggregated free zeaxanthin $(P=0 \cdot 064)$. It remains unclear whether the inclusion of further participants would have allowed to reach statistical significance. The enhanced bioavailability of zeaxanthin from the J-aggregated formulation is suggested to be caused by either the more loosely coupled J-aggregated formulation, by the acylation with two palmitoyl moieties or most likely by a combined effect of both. While zeaxanthin $\mathrm{H}$-aggregates are known to be stabilised by intermolecular hydrogen bonds, forming tightly associated helical arrangements, J-aggregates of zeaxanthin dipalmitate are supposed to form a less tightly coupled liquid-crystalline mesophase $^{(9)}$, presumably enhancing liberation and dissolution of zeaxanthin during digestion. In addition, a presumably differential solubility of zeaxanthin and zeaxanthin dipalmitate in the co-consumed dietary lipids might have also contributed to the enhanced bioavailability of the latter. However, previous studies on the influence of esterification on carotenoid bioavailability had reported only small effects ${ }^{(22-24)}$. For instance, in line with this finding, Chung et al. ${ }^{(23)}$ reported the post-prandial bioavailability of a single dose of a crystalline suspension of free lutein in oil $(10.5 \mu \mathrm{mol} / \mathrm{dose}=6 \mathrm{mg}$ free lutein $/$ dose $)$ to be $10 \%$ higher than that of a single dose of a crystalline suspension of mixed lutein esters in oil $(9.8 \mu \mathrm{mol} /$ dose equivalent to $5.5 \mathrm{mg}$ free lutein/dose), although the difference did not reach statistical significance in only ten subjects $(P>0.05)$.

This study was performed in a cross-over design, which enables a minimisation of effects caused by inter-individual differences in carotenoid absorption. Moreover, the formulation of zeaxanthin and zeaxanthin dipalmitate into identical encapsulation matrices allowed to investigate the effects of carotenoid deposition without further matrix-derived effects. However, the comparison of an esterified $v$. a non-esterified carotenoid did not allow referring the observed differences in absorption to the difference in aggregate form alone. Thus, in agreement with the majority of previous studies, we conclude that the combined effect of aggregation form and esterification represents a rather small effect $( \pm 10-25 \%)$ on the zeaxanthin bioavailability in humans, particularly when compared with other effects such as the addition of lipids or dietary fibre. For instance, the postprandial absorption of lutein from salad was increased by 410 and $570 \%$ by the co-consumption of $150 \mathrm{~g}$ of avocado and $24 \mathrm{~g}$ of avocado oil, respectively ${ }^{(1)}$, whereas the addition of dietary fibre led to a $40-70 \%$ reduction of the post-prandial bioavailability of a lutein supplement ${ }^{(25)}$.

\section{In vitro bioaccessibility}

In vitro bioaccessibility models are widely used to estimate the bioavailability of carotenoids, as they represent low-cost and time-efficient alternatives to human intervention studies. However, direct comparisons of human bioavailability with in vitro bioaccessibility data are rare. Therefore, we subjected the test foods used in the human study to a concurrent in vitro digestion procedure following the standardised INFOGEST model ${ }^{(19)}$ with additional use of cholesterol esterase. Carotenoid liberation was significantly higher when digesting the test food containing the J-aggregated zeaxanthin dipalmitate than when digesting the $\mathrm{H}$-aggregated zeaxanthin. Unexpectedly, the transfer of the liberated carotenoids into the micelles was in reverse order - that is, the bioaccessibility from the test meal with J-aggregated zeaxanthin dipalmitate was lower compared with the test meal with $\mathrm{H}$-aggregated zeaxanthin. In vivo results from our human intervention show that almost exclusively free zeaxanthin occurred in TRL samples, suggesting a hydrolysis of zeaxanthin dipalmitate during digestion. Such hydrolysis was not observed in the procedure based on the INFOGEST model - that is, almost exclusively zeaxanthin dipalmitate was found in the simulated duodenal fluid. The insufficient ester hydrolysis represents a severe disadvantage of the current digestion models when applied to test foods containing carotenoid esters, as such insufficient hydrolysis was not observed in vivo. Missing conversion of strongly hydrophobic carotenoid esters into slightly bipolar free xanthophylls can result in poor micellisation rates as observed in our study. In agreement, earlier simulated digestion experiments have shown that free zeaxanthin is more efficiently incorporated into micelles than zeaxanthin esters ${ }^{(2)}$. The low in vitro bioaccessibility of zeaxanthin from the J-aggregated zeaxanthin dipalmitate test meal is thus likely explained by the inefficient cleavage of zeaxanthin esters in the simulated intestinal phase. Besides zeaxanthin, other xanthophyll esters such as cryptocapsin esters were also shown to be only poorly cleaved during in vitro digestions ${ }^{(27)}$. Consistent with our results, the intake of cryptocapsin esters revealed that only free cryptocapsin had appeared in the TRL fractions, suggesting an efficient cleavage during in vivo absorption ${ }^{(28)}$.

A solution for the lack in xanthophyll ester hydrolysis has not been found to date. Beyond the INFOGEST digestion protocol, we added porcine cholesterol esterase to our intestinal phase of the in vitro digestion, because Breithaupt et al. ${ }^{(29)}$ reported porcine cholesterol esterase to catalyse the hydrolysis of zeaxanthin and lutein esters in vitro. However, this enzyme showed a poor activity towards the extremely apolar zeaxanthin dipalmitate. Likewise, Chitchumroonchokchai \& Failla ${ }^{(26)}$ reported 
the hydrolysis rate to be only $30 \%$ for zeaxanthin esters from a goji berry extract dissolved in olive oil when incubated with bovine cholesterol esterase for $3 \mathrm{~h}$. Therefore, an efficient tool to allow intestinal carotenoid hydrolysis is urgently needed when digesting xanthophyll esters.

\section{Occurrence of esterified zeaxanthin in TAG-rich lipoprotein fractions}

Although traces of lutein monopalmitate have previously been observed in the serum of participants who had consumed a comparably high dose of a mixed lutein ester extract from marigold (corresponding to $15 \mathrm{mg}$ free lutein/d) over 4 months $^{(30)}$, we now report the momentary post-prandial occurrence of zeaxanthin dipalmitate in TRL fractions after a single dose of both free zeaxanthin and zeaxanthin dipalmitate from a food supplement. The zeaxanthin dipalmitate only occurred at single time points and was absent before and after this specific time point. This rare occurrence of zeaxanthin dipalmitate was noted in participants with a comparably high absorption efficiency - that is, with an AUC above the median AUC for post-prandial zeaxanthin bioavailability. As the ester occurred after both ingestion of free and esterified zeaxanthin powder, its origin by the absorption of zeaxanthin dipalmitate from the formulation can be excluded. We support the hypothesis that the presence of zeaxanthin dipalmitate was caused by an in vivo re-esterification process of absorbed zeaxanthin, as has been previously proposed by Granado et $a l .{ }^{(30)}$. As a peak in zeaxanthin dipalmitate occurred in six out of nine times several hours (4-8h) after meal consumption, hepatic zeaxanthin esterification and secretion through VLDL particles appearing in the isolated TRL fraction might represent an overflow route to avoid excessively high concentrations of free zeaxanthin in liver, serum or other unknown compartments. As esterification increases the molecule's hydrophobicity, it might affect the transfer and distribution of zeaxanthin to tissues and other lipoproteins ${ }^{(31)}$. However, the exact localisation of the re-esterification remains unknown and requires further study, particularly because, in three out of the nine instances, the peak was observed also in the 2- or 3-h sample. Moreover, further study on the post-prandial distribution of zeaxanthin into the different lipoprotein fractions should be encouraged to provide further insights into the eventual re-esterification of zeaxanthin.

In conclusion, in the present cohort of healthy non-obese men and women, the bioavailability of J-aggregated zeaxanthin dipalmitate was only marginally higher $(23 \%)$ than that of H-aggregated zeaxanthin $(P=0.064)$. Thus, the aggregation form (H- or J-type) and the presence of zeaxanthin as ester or as free form are suggested to be less important when compared with other well-known influence factors of bioavailability such as the promoting effect of lipid addition or the suppressing effect of added dietary fibre. The INFOGEST in vitro bioaccessibility model supplemented with cholesterol esterase indicated a reverse trend - that is, a higher bioaccessibility of $\mathrm{H}$-aggregated zeaxanthin than that of J-aggregated zeaxanthin dipalmitate. This may be explained by the lack of ester hydrolysis. An efficient enzyme catalysing the hydrolysis of zeaxanthin esters in vitro is currently unavailable but urgently needed for in vitro digestions of test meals containing zeaxanthin esters and, possibly, also other carotenoid esters.

\section{Acknowledgements}

The authors gratefully acknowledge the technical assistance of Julia Senge, Franziska Hägele, Alessa Nas and Karin Scholten during the clinical trial.

This study was funded by the German Research Foundation (Deutsche Forschungsgemeinschaft, project no.: SCHW 1759/2-1). The German Research Foundation had no role in the design, analysis or writing of this article. One of the authors (Judith H.) has been supported by a scholarship by the Studienstiftung des deutschen Volkes.

The authors' contributions are as follows: Judith H., R. C. and R. M. S. have designed the research. Judith H., A. F., M. F., A. B.-W. and R. M. S. have conducted the research. Judith H. has enrolled the participants, analysed the data, written the manuscript and designed figures/tables. Judith $\mathrm{H}$. and Josef $\mathrm{H}$. have carried out the statistical analyses. A. B.-W., R. C., J. H. and R. M. S. have revised the manuscript and substantially contributed to the discussion. All authors have read and approved the final manuscript.

After completion of the clinical study, one of the authors (R. M. S.) has taken up an R\&D position at DSM Nutritional Products (Kaiseraugst, Switzerland), a manufacturer of carotenoid formulations and supplements.

\section{References}

1. Unlu NZ, Bohn T, Clinton SK, et al. (2005) Carotenoid absorption from salad and salsa by humans is enhanced by the addition of avocado or avocado oil. J Nutr 135, 431-436.

2. Brown MJ, Ferruzzi MG, Nguyen ML, et al. (2004) Carotenoid bioavailability is higher from salads ingested with full-fat than with fat-reduced salad dressings as measured with electrochemical detection. Am J Clin Nutr 80, 396-403.

3. Roodenburg AJC, Leenen R, Van Het Hof KH, et al. (2000) Amount of fat in the diet affects bioavailability of lutein esters but not of a-carotene, $ß$-carotene, and vitamin $\mathrm{E}$ in humans. Am J Clin Nutr 71, 1187-1193.

4. Kopec RE, Cooperstone JL, Schweiggert RM, et al. (2014) Avocado consumption enhances human postprandial provitamin a absorption and conversion from a novel high- $\beta$-carotene tomato sauce and from carrots. J Nutr 144, 1158-1166.

5. Schweiggert RM, Kopec RE, Villalobos-Gutierrez MG, et al. (2014) Carotenoids are more bioavailable from papaya than from tomato and carrot in humans: a randomised crossover study. Br J Nutr 111, 490-498.

6. Bernstein PS, Li B, Vachali PP, et al. (2016) Lutein, zeaxanthin, and meso-zeaxanthin: the basic and clinical science underlying carotenoid-based nutritional interventions against ocular disease. Prog Retin Eye Res 50, 34-66.

7. Johnson EJ (2012) A possible role for lutein and zeaxanthin in cognitive function in the elderly. Am J Clin Nutr 96, 1161S-1165s.

8. Mares J (2016) Lutein and zeaxanthin isomers in eye health and disease. Annu Rev Nutr 36, 571-602.

9. Hempel J, Schädle CN, Leptihn S, et al. (2016) Structure related aggregation behavior of carotenoids and carotenoid esters. J Photochem Photobiol A Chem 317, 161-174. 
10. Billsten HH, Sundström V \& Polívka T (2005) Self-assembled aggregates of the carotenoid zeaxanthin: time-resolved study of excited states. J Phys Chem A 109, 1521-1529.

11. Schweiggert RM, Steingass CB, Heller A, et al. (2011) Characterization of chromoplasts and carotenoids of red- and yellow-fleshed papaya (Carica papaya L.). Planta 234, 1031-1044.

12. Hempel J, Schädle CN, Sprenger J, et al. (2017) Ultrastructural deposition forms and bioaccessibility of carotenoids and carotenoid esters from goji berries (Lycium barbarum L.). Food Chem 218, 525-533.

13. Borel P, Grolier P, Mekki N, et al. (1998) Low and high responders to pharmacological doses of $ß$-carotene: Proportion in the population, mechanisms involved and consequences on ß- carotene metabolism. J Lipid Res 39, 2250-2260.

14. Borel P, Desmarchelier C, Nowicki M, et al. (2014) Interindividual variability of lutein bioavailability in healthy men: Characterization, genetic variants involved, and relation with fasting plasma lutein concentration. Am J Clin Nutr 100, $168-175$.

15. US Department of Agriculture (2015) USDA National Nutrient Database for Standard Reference, release 28. http://www.ars. usda.gov/ba/bhnrc/ndl (accessed December 2016).

16. Kopec RE, Schweiggert RM, Riedl KM, et al. (2013) Comparison of high-performance liquid chromatography/tandem mass spectrometry and high-performance liquid chromatography/photo-diode array detection for the quantitation of carotenoids, retinyl esters, $\alpha$-tocopherol and phylloquinone in chylomicron-rich fractions of human plasma. Rapid Commun Mass Spectrom 27, 1393-1402.

17. Khachik F (2003) Process for extraction and purification of lutein, zeaxanthin and rare carotenoids from marigold flowers and plants. Patent US 7173145b, University of Maryland.

18. Britton G (1995) UV/visible spectroscopy. In Carotenoids. Volume 1B: Spectroscopy, pp. 13-62 [G Britton, S LiaaenJensen and $\mathrm{H}$ Pfander, editors]. Basel, Boston, MA, Berlin: Birkhäuser Verlag.

19. Minekus M, Alminger M, Alvito P, et al. (2014) A standardised static in vitro digestion method suitable for food-an international consensus. Food Funct 5, 1113-1124.

20. Schweiggert RM, Mezger D, Schimpf F, et al. (2012) Influence of chromoplast morphology on carotenoid bioaccessibility of carrot, mango, papaya, and tomato. Food Chem 135 , 2736-2742.

21. Aschoff JK, Rolke CL, Breusing N, et al. (2015) Bioavailability of $\beta$-cryptoxanthin is greater from pasteurized orange juice than from fresh oranges - a randomized cross-over study. Mol Nutr Food Res 59, 1896-1904.

22. Landrum J, Bone R, Mendez V, et al. (2012) Comparison of dietary supplementation with lutein diacetate and lutein: A pilot study of the effects on serum and macular pigment. Acta Biochim Pol 59, 167-169.

23. Chung HY, Rasmussen HM \& Johnson EJ (2004) Lutein bioavailability is higher from lutein-enriched eggs than from supplements and spinach in men. J Nutr 134, 1887-1893.

24. Norkus EP, Norkus KL, Dharmarajan TS, et al. (2010) Serum lutein response is greater from free lutein than from esterified lutein during 4 weeks of supplementation in healthy adults. J Am Coll Nutr 29, 575-585.

25. Riedl J, Linseisen J, Hoffmann J, et al. (1999) Some dietary fibers reduce the absorption of carotenoids in women. J Nutr 129, 2170-2176.

26. Chitchumroonchokchai C \& Failla ML (2006) Hydrolysis of zeaxanthin esters by carboxyl ester lipase during digestion facilitates micellarization and uptake of the xanthophyll by Caco-2 human intestinal cells. J Nutr 136, 588-594.

27. Chacón-Ordóñez T, Esquivel P, Jiménez VM, et al. (2016) Deposition form and bioaccessibility of keto-carotenoids from mamey sapote (Pouteria sapota), red bell pepper (Capsicum annuum), and sockeye salmon (Oncorbynchus nerka) filet. J Agric Food Chem 64, 1989-1998.

28. Chacón-Ordóñez T, Schweiggert RM, Bosy-Westphal A, et al. (2017) Carotenoids and carotenoid esters of orange- and yellow-fleshed mamey sapote (Pouteria sapota (Jacq.) H.E. Moore \& Stearn) fruit and their post-prandial absorption in humans. Food Chem 221, 673-682.

29. Breithaupt DE, Bamedi A \& Wirt U (2002) Carotenol fatty acid esters: easy substrates for digestive enzymes? Comp Biochem Physiol B Biochem Mol Biol 132, 721-728.

30. Granado F, Olmedilla B, Gil-Martínez E, et al. (1998) Lutein ester in serum after lutein supplementation in human subjects. Br J Nutr 80, 445-449.

31. Furr HC \& Clark RM (1997) Intestinal absorption and tissue distribution of carotenoids. J Nutr Biochem 8, 364-377. 